\title{
Dynamic Morphologies and Stability of Droplet Interface Bilayers
}

\author{
Benjamin Guiselin, ${ }^{1}$ Jack O. Law, ${ }^{1}$ Buddhapriya Chakrabarti, ${ }^{2}$ and Halim Kusumaatmaja ${ }^{1, *}$ \\ ${ }^{1}$ Department of Physics, Durham University, Durham DH1 3LE, United Kingdom \\ ${ }^{2}$ Department of Physics and Astronomy, University of Sheffield, Sheffield S3 7RH, United Kingdom
}

(Received 24 July 2017; revised manuscript received 24 January 2018; published 6 June 2018)

\begin{abstract}
We develop a theoretical framework for understanding dynamic morphologies and stability of droplet interface bilayers (DIBs), accounting for lipid kinetics in the monolayers and bilayer, and droplet evaporation due to imbalance between osmotic and Laplace pressures. Our theory quantitatively describes distinct pathways observed in experiments when DIBs become unstable. We find that when the timescale for lipid desorption is slow compared to droplet evaporation, the lipid bilayer will grow and the droplets approach a hemispherical shape. In contrast, when lipid desorption is fast, the bilayer area will shrink and the droplets eventually detach. Our model also suggests there is a critical size below which DIBs can become unstable, which may explain experimental difficulties in miniaturizing the DIB platform.
\end{abstract}

DOI: 10.1103/PhysRevLett.120.238001

Droplet interface bilayers (DIBs) are constructed by bringing together two (or more) lipid monolayer-encased water droplets submerged in oil [1,2]. As the droplets contact one another, lipid bilayers form spontaneously. The lipids can be introduced in the bulk of the oil phase [lipid out, Fig. 1(a)] or inside the water droplets [lipid in, Fig. 1(b)]. DIBs can be assembled in several ways, including connecting millimeter-sized aqueous droplets using pipettes, electrodes or lasers [1-4], high throughput microfluidic devices [5-8], and 3D printing [9].

DIBs have a number of advantages over other lipid bilayer platforms. Electrical characterization across the bilayer is easy to perform [10-12]. It is possible to introduce asymmetric bilayers using the lipid-in method $[13,14]$ and to construct complex droplet networks [15-17]. A number of membrane proteins have also been successfully reconstituted across DIBs, including the viral potassium channel, the light-driven proton pump bacteriorhodopsin, and the mechanosensitive channel of large conductance [17-20]. Given these advantages, the potential applications of DIBs are wide ranging, from droplet arrays for ion channel screening [19,20] and chemical microreactors [21,22] to responsive materials $[9,23,24]$ and mimics of electrical circuits and logic gates [25].

The stability of DIBs, however, remains a major issue $[3,26,27]$, especially for DIBs below several hundreds of microns [26]. Furthermore, when DIBs become unstable,

Published by the American Physical Society under the terms of the Creative Commons Attribution 4.0 International license. Further distribution of this work must maintain attribution to the author(s) and the published article's title, journal citation, and DOI. their morphological evolution is extremely rich [27]. As the droplets shrink due to evaporation, the lipid bilayer can (i) zip and increase in size, (ii) unzip until the droplets eventually detach, or (iii) the system can shrink almost uniformly. Here we provide a theoretical framework to address both the issues of DIB stability and dynamic morphologies, for which there has been no explanation to date. The ingredients of our model are the balance between Laplace and osmotic pressures, which determine the evaporation rate of DIBs, and lipid kinetics, which include lipid adsorption, desorption, and exchange between the mono- and bilayers.

Our main results are as follows. First, we are able to construct comprehensive phase diagrams, which reproduce and distinguish the dynamic regimes observed in experiments. Second, our theory predicts a size limit of stable DIBs. For the typical materials used in Refs. [26,27], DIBs smaller than $O(100 \mu \mathrm{m})$ can become unstable. Third, we elucidate a mechanistic understanding for both the dynamic morphology diagram and DIB stability, arising out of a competition between four characteristic timescales: lipid desorptions for the (i) monolayers and (ii) bilayers,

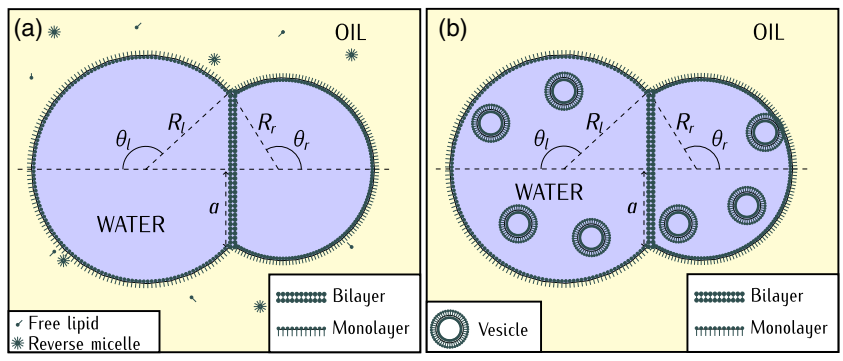

FIG. 1. Schematic diagrams of (a) lipid-out and (b) lipid-in droplet interface bilayers (DIBs). 
(iii) droplet evaporation, and (iv) lipid exchange between the mono- and bilayers.

We begin by describing our model for lipid kinetics. We use subscripts $i=l, r$ to represent the left and right droplets (see Fig. 1), superscript $b$ to label the bilayer, and no superscript for the monolayers (for brevity). The change in the number of lipids on the monolayers and bilayer is due to three different processes. The first process is lipid adsorption from the bulk liquid to the interface:

$$
\frac{d}{d t}\left(\Gamma_{i} S_{i}\right)_{\mathrm{ad}}=k_{\mathrm{on}}\left(\Gamma_{\infty}-\Gamma_{i}\right) S_{i}
$$

The adsorption rate is proportional to the density of available sites per unit area $\left(\Gamma_{\infty}-\Gamma_{i}\right) . \Gamma_{\infty}$ is the density of total available sites, while $\Gamma$ is the density of occupied sites. $k_{\text {on }}$ is the rate constant of lipid adsorption, and $S$ is the monolayer area.

The second process is lipid desorption from the interface to the bulk liquid,

$$
\frac{d}{d t}\left(\Gamma_{i} S_{i}\right)_{\mathrm{de}}=k_{\mathrm{off}} \Gamma_{i} S_{i}
$$

with $k_{\text {off }}$ the desorption rate constant. In general, $k_{\text {on }}$ and $k_{\text {off }}$ depend on the lipid density in the bulk liquid [28]. There are several possible underlying molecular mechanisms for lipid transfer, including rupture and extraction mechanisms [29]. However, in our minimal model, it is neither necessary nor possible to make an explicit statement about molecular mechanisms. For simplicity, we assume $k_{\text {on }}$ and $k_{\text {off }}$ to be constants.

The third process is the exchange of lipids from the monolayer to the bilayer, and vice versa:

$$
\frac{d}{d t}\left(\Gamma_{i} S_{i}\right)_{m b}=\frac{2 \pi a \xi}{k_{B} T}\left[\mu^{b}\left(\Gamma^{b}\right)-\mu_{i}\left(\Gamma_{i}\right)\right]
$$

We assume the current to be proportional to the difference in chemical potentials for the lipids in the monolayers and bilayer, with a proportionality constant $\xi$. The prefactor $2 \pi a$ corresponds to the contact line perimeter where the monolayers meet the bilayer, with $a$ the bilayer radius and $A=\pi a^{2}$ the bilayer area. Here, $k_{B}$ is the Boltzmann constant and $T$ is the system temperature.

The chemical potential of the monolayer can be calculated using the Gibbs-Duhem equation [30], $-\Gamma d \mu=d \gamma$. We employ a standard relation between the monolayer tension and its surface excess [31], $\gamma_{i}\left(\Gamma_{i}\right)=\gamma_{0}+k_{B} T \Gamma_{\infty} \ln \left(1-\Gamma_{i} / \Gamma_{\infty}\right) . \quad \gamma_{0}$ is the surface tension of a clean oil-water interface, when there is no adsorbed lipid. Substituting this equation to the GibbsDuhem equation leads to the following relation for the chemical potential:

$$
\mu_{i}=k_{B} T \ln \left[\Gamma_{i} /\left(\Gamma_{\infty}-\Gamma_{i}\right)\right] .
$$

Similar to Eqs. (1)-(3), equivalent relations for lipid adsorption, desorption, and exchange can be written for the bilayer, as given in the Supplemental Material (SM) [32]. For the lipid-out method, there is no direct lipid exchange from the bulk liquid to the bilayer. The variation in the number of lipids is only due to the exchange between the monolayers and bilayer. Additionally, we assume the bilayer to be incompressible with constant chemical potential $\mu^{b}$ and lipid density $\Gamma^{b}$. Changes in the total number of lipids in the bilayer thus necessarily involve changes in the bilayer area. To justify this assumption, we note that bending deformation to the bilayer is not appreciable in the typical experiments [26,27]. Using the DOPC (1,2dioleoyl-sn-glycero-3-phosphocholine) bilayer as an example, the compressibility is $\approx 290 \mathrm{mN} \mathrm{m}^{-1}$ while its bending modulus is $\approx 29 k_{B} T$ [50]. Consequently, for an energy scale of order of the bending energy, the relative change in lipid density on the bilayer due to compression is negligible, of order $0.01 \%$. Indeed, for higher compression, the lipid bilayer buckles [26,27].

To model droplet evaporation, here we focus on cases where the two droplets forming the DIBs are exactly or close to being symmetric. Thus, evaporation is driven by the imbalance between the osmotic pressure difference $\Delta \Pi$ and the Laplace pressure $P^{L}$ between outside and inside the droplets. The osmotic pressure difference and the Laplace pressure between the two droplets themselves are negligible. The outward flux of water from droplet $i$ can be written as [51]

$$
J_{i}^{\text {out }}=-\frac{d V_{i}}{d t}=\frac{p_{f} v_{w}}{R_{G P} T}\left(\Delta \Pi_{i}-P_{i}^{L}\right) S_{i} .
$$

$v_{w}$ is water's molar volume, $R_{G P}$ is the gas constant, and $p_{f}$ is the monolayer's water permeability coefficient. The osmotic pressure outside the droplets $\Pi^{\text {out }}$ is taken to be constant with time, while inside the droplets we use van 't Hoff's law [30] for dilute solutions, $\Pi_{i}^{\text {in }}=$ $C_{i}^{\text {in }} V_{i}(0) R_{G P} T / V_{i}$, with $C_{i}^{\text {in }}$ the inside osmolarity and $V_{i}(0)$ the initial droplet volume. The Laplace pressure is $P_{i}^{L}=2 \gamma_{i}\left(\Gamma_{i}\right) / R_{i}$, where $R_{i}$ is the radius of droplet $i$; see Fig. 1.

Recent experiments demonstrate that as DIBs become unstable, their dynamic morphologies can follow several distinct pathways. Mruetusatorn et al. [27] reported three classes of behavior. Class I ("bilayer expansion") is highlighted by the observation that the bilayer area grows [Fig. 2(a)] and the droplets' polar angles decrease [Fig. 2(b)] upon evaporation. The polar angles, $\theta_{l}$ and $\theta_{r}$, are defined in Fig. 1. In contrast, for class III ("unzipping"), the bilayer area shrinks [Fig. 2(e)] while the polar angles increase [Fig. 2(f)] until eventually the two droplets detach. Finally, in class II, the DIB shrinks while maintaining approximately constant polar angles (see SM [32], 


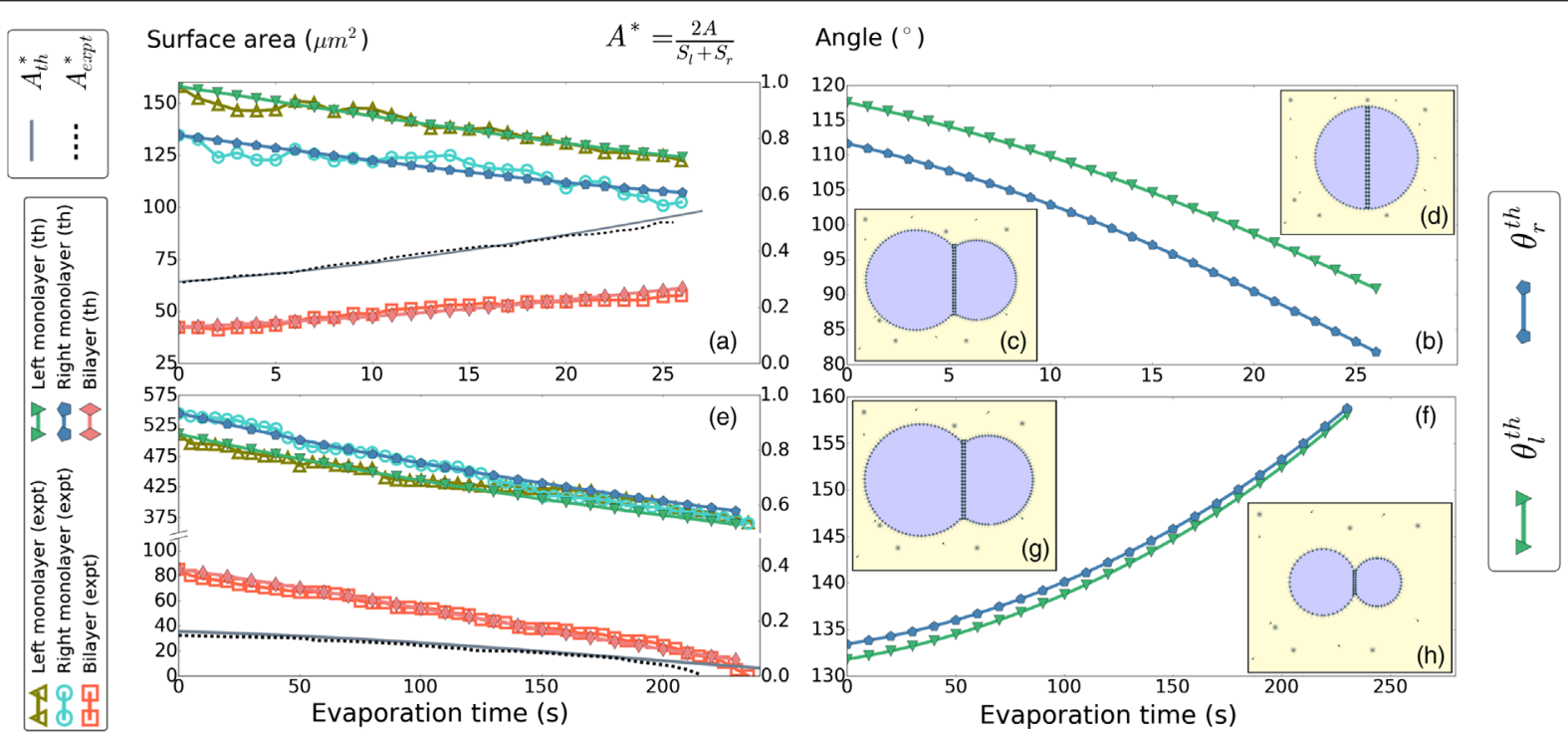

FIG. 2. (a),(e) Surface areas and bilayer area fraction as a function of time superposed with experimental data [27] for the "bilayer expansion" and "unzipping" modes, where the bilayer area expands and shrinks, respectively. (b),(f) In the bilayer expansion (unzipping) mode, this is accompanied by a decrease (increase) in the polar angle. The insets show the initial DIB configurations (c), (g) and their configurations upon evaporation $(\mathrm{d})$, $(\mathrm{h})$. The best-fit parameters to the experimental data are $C_{l / r}^{\text {in }}=$ $(5.11 \pm 0.07) \times 10^{-2} \mathrm{~mol} \mathrm{~m}^{-3}, \Pi^{\text {out }}=(3.64 \pm 0.03) \times 10^{4} \mathrm{~Pa}, k_{\text {off }}=(4.78 \pm 0.04) \times 10^{-3} \mathrm{~s}^{-1}, \Gamma(0)=1.42 \times 10^{18} \mathrm{~m}^{-2}$, and $\mu^{b}=$ $(7.43 \pm 0.02) \times 10^{-2} k_{B} T$ for $\quad(\mathrm{a})-(\mathrm{d}), \quad$ and $\quad C_{l}^{\mathrm{in}}=(1.74 \pm 0.01) \times 10^{-2} \mathrm{~mol} \mathrm{~m}^{-3}, \quad C_{r}^{\mathrm{in}}=(0.72 \pm 0.01) \times 10^{-2} \mathrm{~mol} \mathrm{~m}^{-3}$, $\Pi^{\text {out }}=(2.19 \pm 0.03) \times 10^{4} \mathrm{~Pa}, \quad k_{\text {off }}=(2.50 \pm 0.01) \times 10^{-3} \mathrm{~s}^{-1}, \Gamma(0)=(1.620 \pm 0.004) \times 10^{18} \mathrm{~m}^{-2}$, and $\mu^{b}=(9.96 \pm 0.07) \times$ $10^{-1} k_{B} T$ for (e)-(h). In all cases we have used $\xi=(9.85 \pm 0.34) \times 10^{10}(\mathrm{~ms})^{-1}$.

including comparison with our model). According to our theory, class II corresponds to one of several possible crossover behaviors between the two dominant dynamic modes: bilayer expansion and unzipping.

Figure 2 demonstrates that our model reproduces experimental results with good agreement. The experimental data were obtained using the lipid-out approach, in which DOPC lipids are introduced in soybean oil (top row) and hexadecane (bottom row). As the droplet evaporates, we find the lipid surface density $\Gamma$ closely approaches the saturated density $\Gamma_{\infty}$. Thus, far from equilibrium, lipid adsorption at the monolayer is very small compared to desorption, $k_{\mathrm{on}}\left(\Gamma_{\infty}-\Gamma_{i}\right) \ll k_{\mathrm{off}} \Gamma_{i}$, and can be neglected. To reduce the number of free parameters, we assume the initial lipid monolayer density is the same for the right and left droplets, and equal to the bilayer lipid density, $\Gamma^{b}=\Gamma(0)$. We also assign typical literature values to the following parameters: $p_{f}=80 \mu \mathrm{m} \mathrm{s}^{-1}$ [52-56], $\Gamma_{\infty}=$ $2.3 \times 10^{18} \mathrm{~m}^{-2}$ [57], and $\gamma_{0}=25$ and $44 \mathrm{mN} \mathrm{m}^{-1}$ for soybean oil-water and hexadecane-water interfacial tensions [33]. The remaining parameters in our theory [ $\xi, k_{\text {off }}$, $\left.\Gamma(0), \mu^{b}, \Pi^{\text {out }}, C_{l, r}^{\mathrm{in}}\right]$ are optimized against experimental data using a Markov chain Monte Carlo method [58]. The details are presented in SM [32]. The best-fit parameters are given in the caption of Fig. 2. They compare well to literature values for similar systems, tabulated in SM [32].

Beyond being able to fit the reported experimental data, an important insight from our theory is that we can explain the key factors determining the observed dynamical pathways. From Eqs. (2), (3), and (5), we identify four characteristic timescales: (i) and (ii) lipid desorptions for the mono- and bilayers, $\tau_{\mathrm{des}}=1 / k_{\mathrm{off}}$ and $\tau_{\mathrm{des}}^{b}=1 / k_{\mathrm{off}}^{b}$, (iii) droplet evaporation, $\tau_{e}=\left(R_{G P} T R\right) /\left(p_{f} v_{w} \Pi^{\text {out }}\right)$, and (iv) lipid exchange from the mono- to bilayer, $\tau_{\text {flow }}=\Gamma_{\infty} R / \xi$. We construct a dynamic morphology diagram in Fig. 3, concentrating on the role of the lipid desorption and droplet evaporation timescales. We vary $\beta_{\mathrm{des}}=\tau_{\mathrm{des}} / \tau_{e}$ and $\beta_{\mathrm{des}}^{b}=\tau_{\mathrm{des}}^{b} / \tau_{e}$ while keeping $\tau_{\text {flow }} / \tau_{e}=$ 0.59 constant. We also neglect lipid adsorption, following the results in Fig. 2, where it is negligible compared to lipid desorption. We observe two dominating regimes. The first one is broadly defined by $\beta_{\text {des }} \gtrsim 1$ and $\beta_{\text {des }}^{b} \gtrsim 1$ : if desorption is slow, to compensate the increase in lipid monolayer density upon evaporation (shrinkage in monolayer area), lipids flow from the monolayer to the bilayer, resulting in bilayer zipping and growth. In contrast, for $\beta_{\text {des }} \lesssim 1$ or $\beta_{\text {des }}^{b} \lesssim 1$, desorption from either the mono- or bilayer is fast enough to tackle the rise in lipid density. As the droplets shrink, the bilayer unzips and the droplets detach.

Between these two dominating behaviors, we observe a crossover regime, which occurs when the evaporation timescale becomes similar to the desorption timescale $\left(\beta_{\mathrm{des}} \approx 1\right.$ or $\left.\beta_{\mathrm{des}}^{b} \approx 1\right)$. Class II behavior reported by Mruetusatorn et al. [27] is one of three possible crossover behaviors between the bilayer expansion and unzipping modes (see SM [32]). Varying the value of $\tau_{\text {flow }} / \tau_{e}$ leads to 


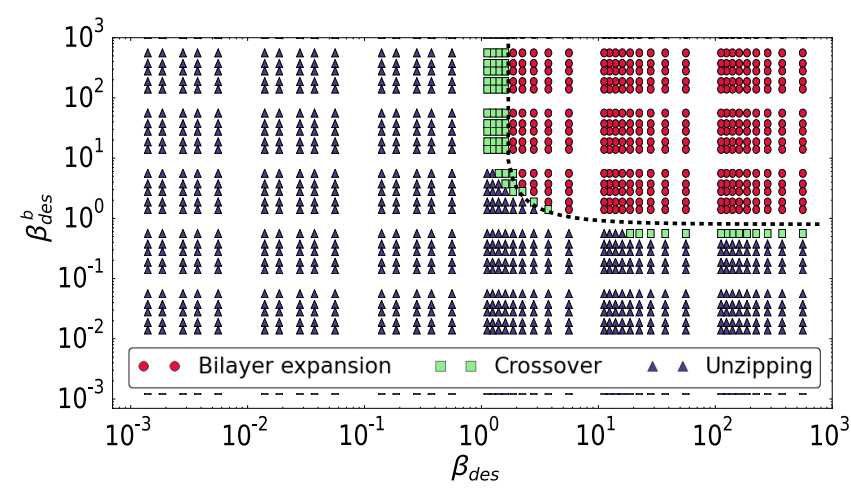

FIG. 3. Dynamic morphology diagram in terms of timescale ratios between lipid desorptions and droplet evaporation, $\beta_{\mathrm{des}}$ and $\beta_{\text {des }}^{b}$. The bilayer expansion mode is observed when desorptions are slow, and the unzipping mode when one of the desorption timescales is fast. The dotted line is a guide to the eye separating these two regions, and the crossover behaviors are detailed in SM [32]. We use $\mu^{b}=0.25 k_{B} T, \Pi^{\text {out }}=2.48 \times 10^{4} \mathrm{~Pa}$, and $C_{l / r}^{\text {in }}=5 \times 10^{-2} \mathrm{~mol} \mathrm{~m}^{-3}$. The other parameters are as in Fig. 2 (top row) for DOPC at soybean oil-water interface.

a similar dynamic morphology diagram, where the bilayer expansion mode is favored at large $\beta_{\mathrm{des}}$ and $\beta_{\mathrm{des}}^{b}$, while the unzipping mode is favored for small $\beta_{\mathrm{des}}$ or $\beta_{\mathrm{des}}^{b}$. With decreasing $\tau_{\text {flow }} / \tau_{e}$, the boundary between the two dominant modes shifts to larger $\beta_{\mathrm{des}}$ and smaller $\beta_{\mathrm{des}}^{b}$ [59].

We now focus on the equilibrium states of DIBs and their relative stability. For simplicity, the left and right droplets are taken to be identical. At equilibrium, lipid adsorption is balanced by desorption. Equating Eqs. (1) and (2) gives equilibrium lipid density, $\Gamma_{i}=\Gamma_{\infty} k_{\mathrm{on}} /\left(k_{\mathrm{on}}+k_{\mathrm{off}}\right)$. Furthermore, the chemical equilibrium between the monoand bilayers imposes the chemical potential of lipids on the bilayer: $\mu^{b}=\mu=k_{B} T \ln \left(k_{\text {on }} / k_{\text {off }}\right)$. Finally, from Eq. (5), the balance between the Laplace and osmotic pressures gives the equilibrium radius of the droplets:

$$
R^{*}=\frac{2}{\Delta \Pi}\left[\gamma_{0}-k_{B} T \Gamma_{\infty} \ln \left(1+\frac{k_{\mathrm{on}}}{k_{\mathrm{off}}}\right)\right] .
$$

Thus, to realize stable DIBs at different sizes for a given set of materials (lipids and oils), it is necessary to adjust the osmotic pressure difference inside and outside the droplets.

Next, starting from the equilibrium states, we perturb the DIB morphologies by randomly varying the outside osmotic pressure, bilayer radius, monolayer lipid densities, and polar angles of the droplets within $\pm 10 \%$ of the initial equilibrium value. In agreement with experimental observations [26], the DIBs are not always stable. Figure 4 shows a transition between stable and unstable DIBs at around $10 \mu \mathrm{m}$ to $1 \mathrm{~mm}$ depending on the polar angle. The simulation parameters are provided in the caption. Each data point is the average behavior from 120 random perturbations [60], and the color intensity signifies the

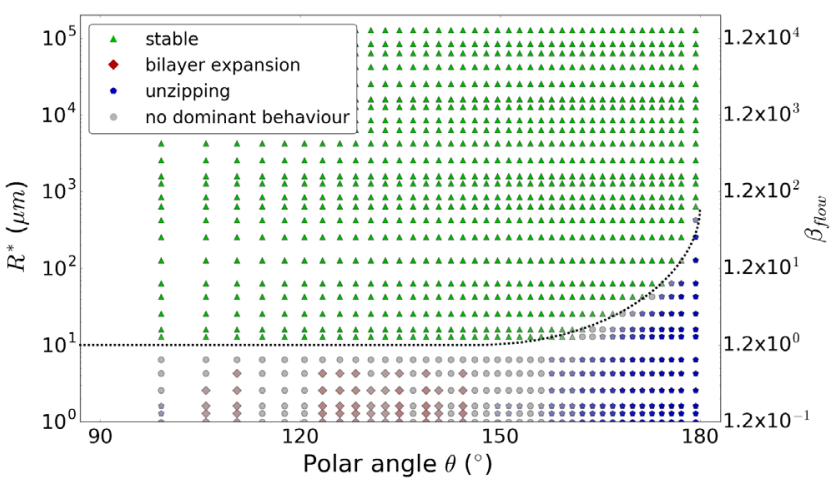

FIG. 4. Stability diagram of DIBs in terms of the droplet radius $R^{*}$ (equivalently $\beta_{\text {flow }}$ ) and polar angle. The dotted lines are guides to the eye. The color intensity for each data point signifies the propensity of the simulation outcomes over 120 runs with random perturbations to the equilibrium configuration. Data points with no dominant behavior (defined as $>55 \%$ of the outcomes) are shown in gray circles. Since we start the simulations close to equilibrium, both lipid adsorption and desorption are relevant. We use $k_{\text {off }}=5 \times 10^{-3} \mathrm{~s}^{-1}$ and $k_{\text {on }}=8 \times 10^{-3} \mathrm{~s}^{-1}$. The other parameters are as in Fig. 2 (top row) for DOPC at soybean oil-water interface.

frequency at which we find (i) the DIB is stable (green triangle), or it destabilizes via (ii) bilayer expansion (red diamond; slightly preferred for polar angle $\lesssim 155^{\circ}$ ), or (iii) unzipping (blue square; preferred for polar angle $\gtrsim 155^{\circ}$ ) mechanisms upon perturbations.

The stability diagram in Fig. 4 can be further understood in terms of timescales. The critical parameter is the ratio between lipid adsorption or desorption and lipid exchange timescales. Here we have set $k_{\text {on }} / k_{\text {off }}=1.6$ and define $\beta_{\text {flow }}=\tau_{\text {flow }} / \tau_{\text {des }}=\Gamma_{\infty} R k_{\text {off }} / \xi$. DIBs are stable when $\beta_{\text {flow }}$ is large. As detailed in SM [32], in the stable regime, the system can reequilibrate without activating lipid flow to the bilayer. In contrast, when the DIBs become unstable for small $\beta_{\text {flow }}$, lipid exchange between the monolayers and bilayer is significant. We observe (detailed in SM [32]) the bilayer expansion mechanism is typically accompanied by net desorption and monolayer to bilayer lipid transfer, while for the unzipping mechanism we have net adsorption and bilayer to monolayer lipid transfer.

To conclude, we have studied the stability of DIBs and the dynamic morphologies they follow when they become unstable. Our theory captures the various dynamic pathways observed in experiments. The bilayer expansion mode is dominant when the timescales for lipid desorptions both in the mono- and bilayers are slow compared to the timescale for droplet evaporation. However, if one of the desorption timescales is fast, either in the monolayers or bilayer, then the unzipping mode is preferred. We also predict the presence of crossover behaviors at the boundary between these two dominant modes in the dynamic morphology diagram. Interestingly some of the timescales identified in our theory are size dependant. This proves to 
be important for the stability of DIBs, where the timescale for lipid exchange between the mono- and bilayers becomes faster for smaller DIBs. This drives instability, and for the experimental systems to which we fitted our model, we predict there is a critical size of order $10 \mu \mathrm{m}$ to $1 \mathrm{~mm}$ below which DIBs can become unstable. An important future work is to exploit this solid theoretical foundation for improving the stability and reproducibility of the DIB platform, which remains a major experimental issue to date, including understanding its possible limit in miniaturization. Another interesting avenue for research is to complement our model with molecular studies on the lipid transfer mechanisms, which determine the rate constants in our model, and consequently the observed dynamic behaviors.

Research data supporting this article can be accessed in Ref. [61].

We thank C. Bain, O. Ces, M. Friddin, Y. Elani, N. Barlow, and G. Bolognesi for useful discussions. We acknowledge funding from EPSRC (EP/J017566/1) and Soft Matter and Functional Interfaces Centre for Doctoral Training (EPSRC EP/L015536/1).

*halim.kusumaatmaja@durham.ac.uk

[1] K. Funakoshi, H. Suzuki, and S. Takeuchi, Anal. Chem. 78, 8169 (2006).

[2] H. Bayley, B. Cronin, A. Heron, M. A. Holden, W. L. Hwang, R. Syeda, J. Thompson, and M. Wallace, Mol. Biosyst. 4, 1191 (2008).

[3] S. Leptihn, O. K. Castell, B. Cronin, E.-H. Lee, L. C. M. Gross, D. P. Marshall, J. R. Thompson, M. Holden, and M. I. Wallace, Nat. Protoc. 8, 1048 (2013).

[4] S. S. Dixit, A. Pincus, B. Guo, and G. W. Faris, Langmuir 28, 7442 (2012).

[5] Y. Elani, A. J. deMello, X. Niu, and O. Ces, Lab Chip 12, 3514 (2012).

[6] S. Thutupalli, J.-B. Fleury, A. Steinberger, S. Herminghaus, and R. Seemann, Chem. Commun. (Cambridge) 49, 1443 (2013).

[7] A. R. Thiam, N. Bremond, and J. Bibette, Langmuir 28, 6291 (2012).

[8] M. A. Czekalska, T. S. Kaminski, S. Jakiela, K. Tanuj Sapra, H. Bayley, and P. Garstecki, Lab Chip 15, 541 (2015).

[9] G. Villar, A. D. Graham, and H. Bayley, Science 340, 48 (2013).

[10] W. L. Hwang, M. A. Holden, S. White, and H. Bayley, J. Am. Chem. Soc. 129, 11854 (2007).

[11] J. L. Poulos, W. C. Nelson, T.-J. Jeon, C.-J. Kim, and J. J. Schmidt, Appl. Phys. Lett. 95, 013706 (2009).

[12] E. C. Freeman, A. B. Farimani, N. R. Aluru, and M. K. Philen, Biomicrofluidics 9, 064101 (2015).

[13] W. L. Hwang, M. Chen, B. Cronin, M. A. Holden, and H. Bayley, J. Am. Chem. Soc. 130, 5878 (2008).

[14] P. J. Milianta, M. Muzzio, J. Denver, G. Cawley, and S. Lee, Langmuir 31, 12187 (2015).
[15] Y. Elani, A. Gee, R. V. Law, and O. Ces, Chem. Sci. 4, 3332 (2013).

[16] B. Schlicht and M. Zagnoni, Sci. Rep. 5, 9951 (2015).

[17] M. A. Holden, D. Needham, and H. Bayley, J. Am. Chem. Soc. 129, 8650 (2007).

[18] H. M. G. Barriga, P. Booth, S. Haylock, R. Bazin, R. H. Templer, and O. Ces, J. R. Soc. Interface 11, 20140404 (2014).

[19] R. Syeda, M. A. Holden, W. L. Hwang, and H. Bayley, J. Am. Chem. Soc. 130, 15543 (2008).

[20] J. L. Poulos, T.-J. Jeon, R. Damoiseaux, E. J. Gillespie, K. A. Bradley, and J. J. Schmidt, Biosens. Bioelectron. 24, 1806 (2009).

[21] Y. Elani, R. V. Law, and O. Ces, Nat. Commun. 5, 5305 (2014).

[22] Y. Elani, X. C. I. Solvas, J. B. Edel, R. V. Law, and O. Ces, Chem. Commun. (Cambridge) 52, 5961 (2016).

[23] S. Punnamaraju, H. You, and A. J. Steckl, Langmuir 28, 7657 (2012).

[24] T. Zhang, D. Wan, J. M. Schwarz, and M. J. Bowick, Phys. Rev. Lett. 116, 108301 (2016).

[25] G. Maglia, A. J. Heron, W. L. Hwang, M. A. Holden, E. Mikhailova, Q. Li, S. Cheley, and H. Bayley, Nat. Nanotechnol. 4, 437 (2009).

[26] J. B. Boreyko, P. Mruetusatorn, S. A. Sarles, S. T. Retterer, and C. P. Collier, J. Am. Chem. Soc. 135, 5545 (2013).

[27] P. Mruetusatorn, J. B. Boreyko, G. A. Venkatesan, S. A. Sarles, D. G. Hayes, and C. P. Collier, Soft Matter 10, 2530 (2014).

[28] J. Eastoe and J. Dalton, Adv. Colloid Interface Sci. 85, 103 (2000).

[29] G. A. Venkatesan, J. Lee, A. B. Farimani, M. Heiranian, C. P. Collier, N. R. Aluru, and S. A. Sarles, Langmuir 31, 12883 (2015).

[30] M. Doi, Soft Matter Physics (Oxford University Press, Oxford, 2013).

[31] J. Liu and U. Messow, Colloid Polym. Sci. 278, 124 (2000).

[32] See Supplemental Material at http://link.aps.org/ supplemental/10.1103/PhysRevLett.120.238001 for additional discussions on the lipid kinetics model for the lipid bilayer, the Markov chain Monte Carlo methods, observed crossover behaviors between "bilayer expansion", and "unzipping" modes, comparison of best-fit parameter values against typical literature values, and further analysis on DIB stability, which includes Refs. [33-49].

[33] H. Zhou, Y. Yao, Q. Chen, G. Li, and S. Yao, Appl. Phys. Lett. 103, 234102 (2013).

[34] G. J. Schütz, H. Schindler, and T. Schmidt, Biophys. J. 73, 1073 (1997).

[35] A. Filippov, G. Orädd, and G. Lindblom, Biophys. J. 84, 3079 (2003).

[36] Z. Derzko and K. Jacobson, Biochemistry 19, 6050 (1980).

[37] P.-M. Gassin, R. Champory, G. Martin-Gassin, J.-F. Dufrêche, and O. Diat, Colloids Surf. A 436, 1103 (2013).

[38] W. F. D. Bennett, J. L. MacCallum, M. J. Hinner, S. J. Marrink, and D. P. Tieleman, J. Am. Chem. Soc. 131, 12714 (2009). 
[39] A. Grafmüller, R. Lipowsky, and V. Knecht, Phys. Chem. Chem. Phys. 15, 876 (2013).

[40] R. Menichetti, K. Kremer, and T. Bereau, Biochem. Biophys. Res. Commun. 498, 282 (2018).

[41] F. Montel, M. Delarue, J. Elgeti, L. Malaquin, M. Basan, T. Risler, B. Cabane, D. Vignjevic, J. Prost, G. Cappello et al., Phys. Rev. Lett. 107, 188102 (2011).

[42] I. Polenz, D. A. Weitz, and J. C. Baret, Langmuir 31, 1127 (2015).

[43] J. J. F. Sleeboom, P. Voudouris, M. T. J. J. M. Punter, F. J. Aangenendt, D. Florea, P. van der Schoot, and H. M. Wyss, Phys. Rev. Lett. 119, 098001 (2017).

[44] S. M. Kirby, S. L. Anna, and L. M. Walker, Langmuir 31, 4063 (2015).

[45] Y. He, P. Yazhgur, A. Salonen, and D. Langevin, Adv. Colloid Interface Sci. 222, 377 (2015).

[46] G. Bleys and P. Joos, J. Phys. Chem. 89, 1027 (1985).

[47] J. K. Ferri, N. Gorevski, C. Kotsmar, M. E. Leser, and R. Miller, Colloids Surf. A 319, 13 (2008).

[48] L. J. Lis, M. McAlister, N. Fuller, R. P. Rand, and V. A. Parsegian, Biophys. J. 37, 667 (1982).

[49] J. N. Israelachvili, Intermolecular and Surface Forces (Academic Press, New York, 2011).

[50] R. M. Venable, F. L. Brown, and R. W. Pastor, Chem. Phys. Lipids 192, 60 (2015).

[51] M. Staykova, M. Arroyo, M. Rahimi, and H. A. Stone, Phys. Rev. Lett. 110, 028101 (2013).

[52] A. Walter and J. Gutknecht, J. Membr. Biol. 90, 207 (1986).
[53] J. C. Mathai, S. Tristram-Nagle, J. F. Nagle, and M. L. Zeidel, J. Gen. Physiol. 131, 69 (2008).

[54] P. J. Milianta, M. Muzzio, J. Denver, G. Cawley, and S. Lee, Langmuir 31, 12187 (2015).

[55] K. Olbrich, W. Rawicz, D. Needham, and E. Evans, Biophys. J. 79, 321 (2000).

[56] A. Finkelstein, J. Gen. Physiol. 68, 127 (1976).

[57] P. Sehgal, H. Doe, and M. Sharma, Colloid Polym. Sci. 282, 188 (2003).

[58] The value of $\xi$ is optimized to fit the experimental data in Fig. 2(a), and its numerical value is kept for other simulations. We only need to fit $\Gamma(0)$ for the hexadecane-water system [Fig. 2(e)], since the availability of experimental surface tension data for the soybean oil-water system allows us to directly determine the initial lipid density.

[59] Consider the case where lipid desorption is faster in the bilayer compared to the monolayers. When lipids can exchange easily from the monolayers to the bilayer, the "bilayer expansion" mode is favored. For the case where lipid desorption is faster in the monolayers compared to the bilayer, flow of lipids from the bilayer to the monolayers will result in bilayer "unzipping."

[60] We have checked that the global shape of the stability diagram and the position of the boundaries between the different domains converge when averaged over more than 100 simulations. The stochasticity displayed in Fig. 4 results from the random initial perturbations, as described in SM [32].

[61] https://doi.org/10.15128/r2mk61rg93b. 